\title{
Clinical and radiographic parameters associated with best versus worst clinical outcomes in minimally invasive spinal deformity surgery
}

\author{
Khoi D. Than, MD, ${ }^{1}$ Paul Park, MD, ${ }^{2}$ Kai-Ming Fu, MD, ${ }^{3}$ Stacie Nguyen, MPH, ${ }^{4}$ \\ Michael Y. Wang, MD, ${ }^{5}$ Dean Chou, MD, ${ }^{6}$ Pierce D. Nunley, MD, ${ }^{7}$ Neel Anand, MD, ${ }^{8}$ \\ Richard G. Fessler, MD, PhD, ${ }^{9}$ Christopher I. Shaffrey, MD, ${ }^{10}$ Shay Bess, MD, ${ }^{11}$ \\ Behrooz A. Akbarnia, MD, ${ }^{4}$ Vedat Deviren, MD, ${ }^{12}$ Juan S. Uribe, MD, ${ }^{13}$ Frank La Marca, MD, ${ }^{2}$ \\ Adam S. Kanter, MD, ${ }^{14}$ David O. Okonkwo, MD, PhD, ${ }^{14}$ Gregory M. Mundis Jr., MD, ${ }^{4}$ \\ Praveen V. Mummaneni, MD, ${ }^{6}$ and the International Spine Study Group
}

1Department of Neurological Surgery, Oregon Health \& Science University, Portland, Oregon; ${ }^{2}$ Department of Neurological Surgery, University of Michigan, Ann Arbor, Michigan; ${ }^{3}$ Weill Cornell Brain and Spine Center, New York, New York; ${ }^{4}$ San Diego Center for Spinal Disorders, La Jolla, California; ${ }^{5}$ University of Miami Spine Institute, Miami, Florida; ${ }^{6}$ Department of Neurological Surgery and ${ }^{12}$ Spine Center, UCSF Medical Center, San Francisco, California; ${ }^{7}$ Spine Institute of Louisiana, Shreveport, Louisiana; ${ }^{8}$ Orthopedic Spine Surgery, Cedars-Sinai, Los Angeles, California; ${ }^{9}$ Department of Neurological Surgery, Rush University Medical Center, Chicago, Illinois; ${ }^{10}$ Department of Neurological Surgery, University of Virginia, Charlottesville, Virginia; ${ }^{11}$ Rocky Mountain Scoliosis \& Spine, Denver, Colorado; ${ }^{13}$ Department of Neurosurgery and Brain Repair, University of South Florida, Tampa, Florida; and ${ }^{14}$ Department of Neurosurgery, University of Pittsburgh, Pennsylvania

OBJECTIVE Minimally invasive surgery (MIS) techniques are increasingly used to treat adult spinal deformity. However, standard minimally invasive spinal deformity techniques have a more limited ability to restore sagittal balance and match the pelvic incidence-lumbar lordosis (PI-LL) than traditional open surgery. This study sought to compare "best" versus "worst" outcomes of MIS to identify variables that may predispose patients to postoperative success.

METHODS A retrospective review of minimally invasive spinal deformity surgery cases was performed to identify parameters in the $20 \%$ of patients who had the greatest improvement in Oswestry Disability Index (ODI) scores versus those in the $20 \%$ of patients who had the least improvement in ODI scores at 2 years' follow-up.

RESULTS One hundred four patients met the inclusion criteria, and the top $20 \%$ of patients in terms of ODI improvement at 2 years (best group, 22 patients) were compared with the bottom 20\% (worst group, 21 patients). There were no statistically significant differences in age, body mass index, pre- and postoperative Cobb angles, pelvic tilt, pelvic incidence, levels fused, operating room time, and blood loss between the best and worst groups. However, the mean preoperative ODI score was significantly higher (worse disability) at baseline in the group that had the greatest improvement in ODI score (58.2 vs $39.7, p<0.001)$. There was no difference in preoperative PI-LL mismatch $\left(12.8^{\circ}\right.$ best vs $19.5^{\circ}$ worst, $p=0.298$ ). The best group had significantly less postoperative sagittal vertical axis (SVA; 3.4 vs $6.9 \mathrm{~cm}, p=0.043$ ) and postoperative PI-LL mismatch $\left(10.4^{\circ}\right.$ vs $\left.19.4^{\circ}, p=0.027\right)$ than the worst group. The best group also had better postoperative visual analog scale back and leg pain scores $(p=0.001$ and $p=0.046$, respectively).

CONCLUSIONS The authors recommend that spinal deformity surgeons using MIS techniques focus on correcting a patient's PI-LL mismatch to within $10^{\circ}$ and restoring SVA to $<5 \mathrm{~cm}$. Restoration of these parameters seems to impact which patients will attain the greatest degree of improvement in ODI outcomes, while the spines of patients who do the worst are not appropriately corrected and may be fused into a fixed sagittal plane deformity.

http://thejns.org/doi/abs/10.3171/2015.12.SPINE15999

KEY WORDS minimally invasive surgery; deformity; spine; outcome

ABBREVIATIONS ASD = adult spinal deformity; BMI = body mass index; EBL = estimated blood loss; $\mathrm{LL}$ = lumbar lordosis; LLIF = lateral lumbar interbody fusion; MIS = minimally invasive surgery; MISDEF = minimally invasive spinal deformity; MI-TLIF = minimally invasive transforaminal lumbar interbody fusion; ODI = Oswestry Disability Index; OR = operating room; PI = pelvic incidence; PI-LL = pelvic incidence-lumbar lordosis; PT = pelvic tilt; SRS = Scoliosis Research Society; SVA = sagittal vertical axis; TK = thoracic kyphosis; VAS = visual analog scale.

SUBMITTED August 20, 2015. ACCEPTED December 9, 2015.

INCLUDE WHEN CITING Published online March 4, 2016; DOI: 10.3171/2015.12.SPINE15999. 
$\mathrm{M}$ INIMALLY invasive surgery (MIS) techniques are increasingly used in spine surgery, including in the treatment of adult spinal deformity (ASD). Such techniques include minimally invasive transpsoas retroperitoneal approaches for lateral lumbar interbody fusion (LLIF) and minimally invasive transforaminal lumbar interbody fusion (MI-TLIF). Previous work has suggested that minimally invasive spinal deformity correction is associated with fewer intraoperative complications than open or hybrid techniques ${ }^{9}$ with comparable clinical outcomes. ${ }^{2}$ However, with the relatively limited anatomical exposure of MIS techniques, there is some concern that such procedures are not well suited to correct severe deformities. Mummaneni and colleagues ${ }^{5}$ recently proposed a minimally invasive spinal deformity (MISDEF) surgery algorithm. This algorithm identifies patients appropriate for minimally invasive spinal deformity correction as those with a sagittal vertical axis (SVA) $\leq 6 \mathrm{~cm}$, pelvic tilt $(\mathrm{PT}) \leq 25^{\circ}$, pelvic incidence-lumbar lordosis (PI-LL) mismatch $\leq 30^{\circ}$, and/or thoracic kyphosis $(\mathrm{TK}) \leq 60^{\circ}$. Conversely, poor candidates for minimally invasive spinal deformity correction are those with a fixed SVA $>6 \mathrm{~cm}$, PT $>25^{\circ}$, PI-LL $>30^{\circ}$, and $/$ or TK $>60^{\circ}$.

In this study, data obtained from a large multicenter database of patients who had undergone MIS for ASD were analyzed. Patients with the "best" clinical outcomes were compared with those who had the "worst" to identify the variables that predict which patients with ASD who undergo MIS will have the best outcomes.

\section{Methods \\ Study Design}

This was a retrospective analysis of data collected from a multicenter database of patients with ASD who had been treated with MIS techniques; data were contributed by 11 participating institutions (each site had institutional review board approval). Inclusion criteria for entry into the multicenter database were patient age $\geq 18$ years, diagnosis of ASD, minimum of 2 years of follow-up, and at least 1 of the following factors: coronal Cobb angle $>20^{\circ}$, SVA $>5 \mathrm{~cm}$, and/or PT $>20^{\circ}$. This database included patients who had only MIS and those who had a hybrid procedure involving an MIS component as well as a traditional open component. The surgical approach for a given patient was used at the discretion of their surgeon. An initial query for patients who had only MIS identified 104 patients. This MIS group consisted of patients who had undergone stand-alone LLIF as well as LLIF and/or MI-TLIF with percutaneous pedicle screw fixation. A schematic example is depicted in Fig. 1.

\section{Data Collection}

Outcomes were assessed based on 2-year postoperative clinical outcome variables, including visual analog scale (VAS) scores for both back and leg and the Oswestry Disability Index (ODI). Demographic and intraoperative data were also recorded for each outcome group, including age, body mass index (BMI), estimated blood loss (EBL), operating room (OR) time, and number of levels treated. All patients had 36-inch standing scoliosis radiographs at baseline and at least 2-years' follow-up. Measured radio- graphic parameters included coronal curve, pelvic incidence (PI), LL, PI-LL mismatch, PT, and SVA. All films were measured at a single location for consistency.

\section{Statistical Analysis}

Best-group patients included the top $20 \%$ of patients in terms of their change in ODI score, while worst-group patients included the bottom $20 \%$ of patients in terms of their change in ODI score. The mean and standard deviation were used to describe continuous variables, and frequency analyses were used for categorical variables. Comparisons between the 2 outcome groups were performed using nonparametric Mann-Whitney U-test and chi-square analysis. The change between preoperative and postoperative parameters was analyzed using a paired Wilcoxon signedrank test. A p value $\leq 0.05$ was considered significant.

\section{Results}

Of the 104 patients who met the inclusion criteria, 22 patients with the greatest improvements in their ODI score were included in the best group, while 21 patients with the least improvement in their ODI score composed the worst group. Average follow-up for the entire study was 32.4 months (best 32.7 months, worst 32.0 months). There were no statistically significant differences between the 2 groups in terms of age, BMI, OR time, EBL, or number of levels fused (Table 1). In terms of baseline radiographic parameters, there were no statistically significant differences between the 2 groups in terms of Cobb angle, PT, PI, LL, PI-LL, or SVA. Similarly, as regards baseline functional scores, there were no statistically significant differences in VAS back or leg pain scores.

There was a statistically significant difference in preoperative ODI scores between the 2 groups. Best-group patients had an average preoperative ODI score of 58.2, while worst-group patients had a preoperative ODI score of 39.7 ( $\mathrm{p}<0.001)$.

There were no statistically significant postoperative differences between the best and worst groups' average Cobb angle (although both groups had a smaller value than preoperatively), PT, PI, or LL. However, there was a statistically significant difference in the postoperative PILL mismatch (best $10.4^{\circ}$ vs worst $19.4^{\circ}, \mathrm{p}=0.027$ ) and SVA (best $34.0 \mathrm{~mm}$ vs worst $68.6 \mathrm{~mm}, \mathrm{p}=0.043$ ).

In terms of postoperative outcomes, there was a statistically significant difference between the groups in ODI scores (best 11.9 vs worst $50.4, \mathrm{p}<0.001$ ). Patients in the best group saw their ODI score improve from 58.2 to 11.9, on average, while patients in the worst group saw their ODI score get worse (from 39.7 to 50.4). There was a statistically significant difference between groups in VAS back pain (best 2.0 vs worst $4.6, p<0.001$ ) and VAS leg pain (best 1.7 vs worst $3.3, p=0.046$ ). It should be noted that these postoperative VAS scores all improved compared with baseline values.

\section{Discussion}

In this study of 104 patients who had undergone MIS for ASD, we found that the patients who had the greatest improvement in their ODI scores were the ones who had 

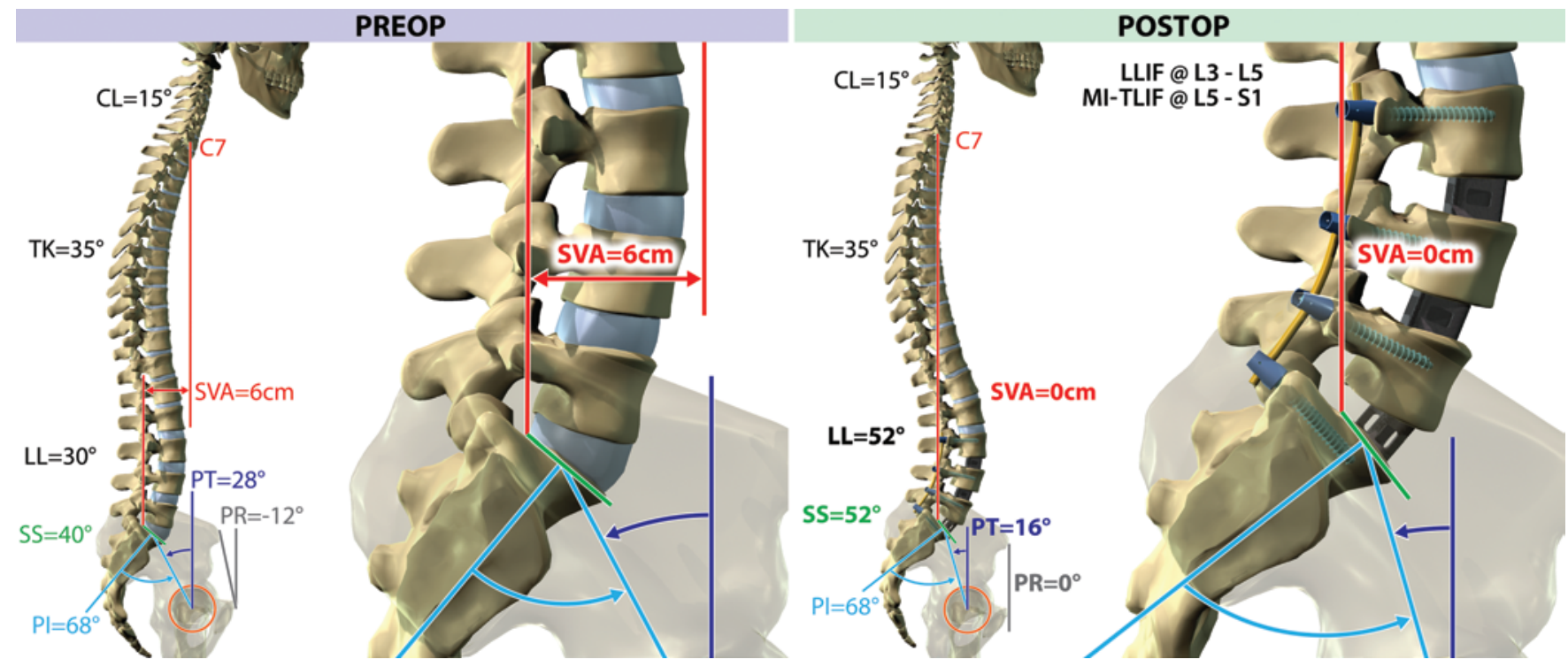

FIG. 1. Schematic of a spine treated with an LLIF at L3-4 and L4-5 and an MI-TLIF at L5-S1. Copyright Kenneth Probst. Published with permission. $\mathrm{CL}=$ cervical lordosis; $\mathrm{SS}=$ sacral slope; $\mathrm{PR}=$ pelvic rotation. Figure is available in color online only.

optimized postoperative spinopelvic parameters. Specifically, patients had SVA $<5 \mathrm{~cm}$ and PI-LL mismatch near $10^{\circ}$. Conversely, patients who had poor outcomes did not have this optimized SVA and PI-LL mismatch. Patients in the best group started with an average SVA of $32.8 \mathrm{~mm}$ that was maintained postoperatively at $34 \mathrm{~mm}$, while patients in the worst group started with an average SVA of $51.3 \mathrm{~mm}$ that worsened to $68.6 \mathrm{~mm}$ postoperatively. Similarly, patients in the best group had an average starting PILL mismatch of $12.8^{\circ}$ that improved to $10.4^{\circ}$ postoperatively, while worst-group patients started with an average PI-LL of $19.5^{\circ}$ that was unchanged to $19.4^{\circ}$ with surgery. These findings underscore the importance of sagittal balance and PI-LL matching in ASD outcomes and suggest that the spines of patients who do the worst are not appropriately corrected in the sagittal plane and are perhaps fused into a fixed sagittal plane deformity.

The increased mean SVA in both groups probably represents the limited ability to improve sagittal alignment with early MIS techniques. Similarly, the unchanged PILL mismatch in the worst group reflects a lack of sagittal alignment improvement obtained by the early MIS approaches we used.

These results highlight the basis of the MISDEF algorithm we created to guide MIS versus open treatment in patients with higher baseline SVA, PT, and PI-LL mismatch who are categorized into Class III (or open) approaches. It is important to note, however, that the patients evaluated in the present study represent early MIS approaches to ASD. Newer techniques, including anterior longitudinal ligament release through an LLIF or anterior LIF approach, have been shown to significantly improve sagittal alignment. In addition, the use of hybrid surgery (where both MIS and open techniques are used) may be a more effective option.

Previous work has clearly demonstrated the importance of these parameters. In a multicenter study, Glassman and colleagues ${ }^{1}$ retrospectively reviewed 752 patients
TABLE 1. Demographics, radiographic parameters, and patient outcomes*

\begin{tabular}{|c|c|c|c|}
\hline Parameter & $\begin{array}{l}\text { Best Group } \\
(\mathrm{n}=22)\end{array}$ & $\begin{array}{l}\text { Worst Group } \\
\qquad(n=21)\end{array}$ & $p$ Value \\
\hline Age (yrs) & $64 \pm 13.1$ & $63.1 \pm 12.1$ & 0.715 \\
\hline BMI & $26.8 \pm 26.8$ & $28.9 \pm 28.9$ & 0.314 \\
\hline OR time (mins) & $419.5 \pm 212.7$ & $473.3 \pm 215.1$ & 0.399 \\
\hline $\mathrm{EBL}(\mathrm{ml})$ & $458.2 \pm 475.6$ & $571.2 \pm 606.0$ & 0.253 \\
\hline Levels fused & $3.7 \pm 1.6$ & $3.8 \pm 2.0$ & 0.757 \\
\hline \multicolumn{4}{|l|}{ Preop } \\
\hline Cobb angle $\left({ }^{\circ}\right)$ & $32.5 \pm 10.8$ & $37 \pm 16.2$ & 0.506 \\
\hline $\mathrm{PT}\left({ }^{\circ}\right)$ & $22.8 \pm 8.9$ & $27.8 \pm 11.8$ & 0.244 \\
\hline $\mathrm{PI}\left({ }^{\circ}\right)$ & $50.4 \pm 9.4$ & $57.4 \pm 11.2$ & 0.087 \\
\hline $\operatorname{LL}\left({ }^{\circ}\right)$ & $37.1 \pm 11.0$ & $37.9 \pm 19.0$ & 0.47 \\
\hline PI-LL $\left({ }^{\circ}\right)$ & $12.8 \pm 11.3$ & $19.5 \pm 20.6$ & 0.298 \\
\hline SVA (mm) & $32.8 \pm 52.1$ & $51.3 \pm 66.6$ & 0.405 \\
\hline ODI & $58.2 \pm 14.3$ & $39.7 \pm 12.5$ & $<0.001$ \\
\hline VAS back pain & $6.7 \pm 2.0$ & $6.6 \pm 1.9$ & 0.768 \\
\hline VAS leg pain & $6.5 \pm 2.5$ & $5.8 \pm 2.8$ & 0.538 \\
\hline \multicolumn{4}{|l|}{ Postop } \\
\hline Cobb angle $\left({ }^{\circ}\right)$ & $21.9 \pm 14.4$ & $21.2 \pm 12.5$ & 0.911 \\
\hline $\mathrm{PT}\left({ }^{\circ}\right)$ & $22.6 \pm 10.2$ & $27.8 \pm 9.5$ & 0.085 \\
\hline $\mathrm{PI}\left({ }^{\circ}\right)$ & $51 \pm 8.9$ & $57.4 \pm 10.8$ & 0.061 \\
\hline $\operatorname{LL}\left({ }^{\circ}\right)$ & $40.4 \pm 10.9$ & $38 \pm 13.7$ & 0.513 \\
\hline PI-LL $\left({ }^{\circ}\right)$ & $10.4 \pm 13.3$ & $19.4 \pm 14.2$ & 0.027 \\
\hline SVA (mm) & $34 \pm 57.2$ & $68.6 \pm 79.6$ & 0.043 \\
\hline ODI & $11.9 \pm 11.7$ & $50.4 \pm 14.8$ & $<0.001$ \\
\hline VAS back pain & $2 \pm 2.2$ & $4.6 \pm 2.5$ & 0.001 \\
\hline VAS leg pain & $1.7 \pm 2.2$ & $3.3 \pm 2.5$ & 0.046 \\
\hline
\end{tabular}

* Values expressed as the mean \pm standard deviation. Boldface type indicates statistical significance. 
with ASD (352 with positive sagittal balance) and found that as SVA increased, numerous measures of health status (specifically the Scoliosis Research Society [SRS] patient questionnaire, Medical Outcomes Study 12-Item Short Form, and ODI) worsened in a linear fashion. Mac-Thiong and colleagues ${ }^{4}$ conducted a similar prospective study in 73 patients and found that an SVA $>6 \mathrm{~cm}$ correlated with a poor ODI score (defined as $>34$ ). Restoring sagittal balance with surgery benefited these patients. In a retrospective study of 35 patients who had undergone pedicle subtraction osteotomy for fixed sagittal imbalance with at least 5 years of follow-up, Kim and colleagues ${ }^{3}$ found that an SVA $<8 \mathrm{~cm}$ was associated with improved SRS outcome scores.

Disability also occurs when PI and LL are not matched to within $10^{\circ}$. In a prospective multicenter study of 492 patients with ASD, Schwab and colleagues ${ }^{6}$ found that in addition to an SVA $>47 \mathrm{~mm}$, disability (defined as ODI $>40$ ) was predicted by a PI-LL $\geq 11^{\circ}$ and PT $\geq 22^{\circ}$. Even patients with PI-LL mismatch in the setting of a normal SVA, which is a compensated sagittal spinopelvic malalignment, can have serious disability and can potentially benefit from surgery. ${ }^{8}$ Clearly, the goals of ASD surgery are to normalize SVA and PI-LL mismatch.

Comparing patients who undergo similar surgical treatment but have very different clinical outcomes provides useful information to surgeons in terms of patient selection and surgical goals. Smith and colleagues ${ }^{7}$ previously compared best and worst clinical outcomes (as measured by SRS-22 and ODI scores) for 227 patients who had undergone open ASD surgery. The strongest predictors of worst final SRS-22 scores included higher baseline SRS-22 scores, depression, and surgical complications. The strongest predictors of worst final ODI scores included higher baseline ODI scores, higher baseline BMI, and greater SVA at the 2-year follow-up. Other factors found to be associated with worst outcomes included patient comorbidities. Similarly, in our study a higher postoperative SVA was found to be a potential predictive variable for worse ODI score. Conversely, a high baseline ODI score was found to be associated with the best final ODI score.

Of great interest to spine surgeons is how outcomes compare after open versus minimally invasive spinal deformity surgery. Data from prior work by the International Spine Study Group may allow for comparison of best outcomes between spinal deformity patients treated with open (Smith et al. study ${ }^{7}$ ) versus MIS (present study) techniques. Open surgery patients with the best outcomes had an ODI improvement of 36 points (score decreased from 43 to 7), whereas MIS patients had an improvement of greater than 46 points (from 58 to 12). Open surgery patients with the best outcomes had a VAS leg pain score improvement of 3.9 points (from 5.1 to 1.2), whereas MIS patients had an improvement of 4.8 points (from 6.5 to 1.7). Lastly, open surgery patients with the best outcomes had a VAS back pain score improvement of 5.7 points (from 7.2 to 1.5), whereas MIS patients had an improvement of 4.7 points (from 6.7 to 2). Although these comparisons do not account for differences in study design or patient characteristics, they do suggest that the results after MIS surgery are at least as good as after open surgery.

This study has several limitations. Its primary limita- tion is its retrospective study design and the data review of a relatively small number of patients from a multicenter study. It is notable that, although the difference was not statistically significant, patients in the best group started with a better SVA and PI-LL mismatch than the patients in the worst group. It is possible that a statistically significant difference could be observed if the standard deviation was not higher than the mean. Future prospective studies involving larger numbers of patients will help to solidify and confirm the findings in this study. In addition, the multicenter nature of the study introduces a level of variability that is difficult to control for with respect to data collection but allows for more generalizable results.

\section{Conclusions}

We recommend that minimally invasive spinal deformity surgeons focus on correcting a patient's PI-LL mismatch to within $10^{\circ}$ and restoring SVA to $<5 \mathrm{~cm}$. Restoration of these parameters seems to impact which patients will achieve the greatest degree of improvement in ODI outcomes, while the spines of patients who fare the worst are not appropriately corrected and are perhaps fused into a fixed sagittal plane deformity.

\section{Acknowledgments}

We thank Shirley McCartney, $\mathrm{PhD}$, for her help with editing the final manuscript. We also thank the AANS/CNS Joint Section of Disorders of the Spine and Peripheral Nerves for financial support.

\section{References}

1. Glassman SD, Bridwell K, Dimar JR, Horton W, Berven S, Schwab F: The impact of positive sagittal balance in adult spinal deformity. Spine (Phila Pa 1976) 30:2024-2029, 2005

2. Haque RM, Mundis GM Jr, Ahmed Y, El Ahmadieh TY, Wang MY, Mummaneni PV, et al: Comparison of radiographic results after minimally invasive, hybrid, and open surgery for adult spinal deformity: a multicenter study of 184 patients. Neurosurg Focus 36(5):E13, 2014

3. Kim YJ, Bridwell KH, Lenke LG, Cheh G, Baldus C: Results of lumbar pedicle subtraction osteotomies for fixed sagittal imbalance: a minimum 5-year follow-up study. Spine (Phila Pa 1976) 32:2189-2197, 2007

4. Mac-Thiong JM, Transfeldt EE, Mehbod AA, Perra JH, Denis F, Garvey TA, et al: Can C7 plumbline and gravity line predict health related quality of life in adult scoliosis? Spine (Phila Pa 1976) 34:E519-E527, 2009

5. Mummaneni PV, Shaffrey CI, Lenke LG, Park P, Wang MY, La Marca F, et al: The minimally invasive spinal deformity surgery algorithm: a reproducible rational framework for decision making in minimally invasive spinal deformity surgery. Neurosurg Focus 36(5):E6, 2014

6. Schwab FJ, Blondel B, Bess S, Hostin R, Shaffrey CI, Smith JS, et al: Radiographical spinopelvic parameters and disability in the setting of adult spinal deformity: a prospective multicenter analysis. Spine (Phila Pa 1976) 38:E803-E812, 2013

7. Smith JS, Shaffrey CI, Lafage V, Schwab F, Scheer JK, Protopsaltis T, et al: Comparison of best versus worst clinical outcomes for adult spinal deformity surgery: a retrospective review of a prospectively collected, multicenter database with 2-year follow-up. J Neurosurg Spine 23:349-359, 2015 
8. Smith JS, Singh M, Klineberg E, Shaffrey CI, Lafage V, Schwab FJ, et al: Surgical treatment of pathological loss of lumbar lordosis (flatback) in patients with normal sagittal vertical axis achieves similar clinical improvement as surgical treatment of elevated sagittal vertical axis: clinical article. J Neurosurg Spine 21:160-170, 2014

9. Uribe JS, Deukmedjian AR, Mummaneni PV, Fu KM, Mundis GM Jr, Okonkwo DO, et al: Complications in adult spinal deformity surgery: an analysis of minimally invasive, hybrid, and open surgical techniques. Neurosurg Focus 36(5):E15, 2014

\section{Disclosures}

Dr. Shaffrey is a consultant for Biomet, Medtronic, NuVasive, $\mathrm{K} 2 \mathrm{M}$, and Stryker; owns stock in NuVasive; and holds patents and receives royalties from Biomet, NuVasive, and Medtronic. Dr. Wang is a consultant for DePuy Synthes Spine, Aesculap Spine, Joimax, and K2M; and holds patents with DePuy Synthes Spine. Dr. Park is a consultant for Globus, Medtronic, and Biomet; and receives royalties from Globus. Dr. Nunley is a consultant for LDR Spine and K2M; holds patents with LDR Spine, Osprey Medical, and K2M; and owns stock in Amedica, Safewire, Paradigm, and Spineology. Dr. Uribe is a consultant for and owns stock in NuVasive. Dr. Okonkwo is a consultant for Biomet. Dr. Bess is a consultant for NuVasive, K2M, and Allosource; holds patents with Innovasis; receives clinical or research support for the study described from DePuy Synthes Spine; and receives support of non-study-related clinical or research effort from Medtronic, K2M, Stryker, and Innovasis. Dr. La Marca is a consultant for Globus and Biomet. Dr. Mundus is a consultant for NuVasive, K2M, Misonix, Medicrea, and Ellipse; receives royalties from NuVasive and $\mathrm{K} 2 \mathrm{M}$; and has a nonfinancial relationship with a person at NuVasive. Dr. Chou is a consultant for Orthofix, Globus, and Medtronic. Dr. Mummaneni is a consultant for DePuy Synthes Spine; owns stock in Spinicity/ISD; receives honoraria from AO Spine and Globus; receives royalties from Thieme Publishing, Springer Publishing, Taylor and Francis Publishing, and DePuy Synthes Spine; and receives a grant from AO Spine. Dr. Anand is a consultant for Medtronic; owns stock in Globus and Medtronic; holds patents with Medtronic; and receives royalties from Medtronic, Globus, and NuVasive. Dr. Deviren is a consultant for NuVasive, Guidepoint, and Stryker.

\section{Author Contributions}

Conception and design: Than, Park, Fu, Nguyen, Mundis, Mummaneni. Acquisition of data: Than, Park, Fu, Wang, Chou, Nunley, Anand, Fessler, Shaffrey, Bess, Akbarnia, Deviren, Uribe, La Marca, Kanter, Okonkwo, Mundis, Mummaneni. Analysis and interpretation of data: Than, Park, Nguyen, Okonkwo, Mundis. Drafting the article: Than, Nguyen,

Mummaneni. Critically revising the article: Than, Park, Nguyen, Mummaneni. Reviewed submitted version of manuscript: Than, Park, Nguyen, Mummaneni. Approved the final version of the manuscript on behalf of all authors: Than. Statistical analysis: Nguyen. Administrative/technical/material support: Nguyen, Bess, Mundis, Mummaneni. Study supervision: Shaffrey, Mundis, Mummaneni.

\section{Correspondence}

Khoi D. Than, Department of Neurological Surgery, Mail Code: CH8N, Oregon Health \& Science University, 3303 S.W. Bond Ave., Portland, OR 97239. email: thank@ohsu.edu. 